This is a pre-print of an article published in: Photonic Network Communications. The final authenticated version is available online at:

https://doi.org/10.1007/s11107-015-0562-7 


\title{
Experimental assessment of a cognitive mechanism to reduce the impact of outdated TEDs in optical networks
}

\author{
R.J. Durán, N. Fernández, D. Siracusa, A. Francescon, I. de Miguel, I. Rodríguez, \\ J.C. Aguado, E. Salvadori and R.M. Lorenzo
}

R.J. Durán, N. Fernández, I. de Miguel, I. Rodríguez, J.C. Aguado and R.M. Lorenzo are with the Universidad de Valladolid, Paseo de Belén 15 , 47011 Valladolid, Spain. (e-mail: rduran@tel.uva.es)

D. Siracusa, A. Francescon and E. Salvadori are with CREATE-NET, Via alla Cascata 56/D, 38123 Trento, Italy (e-mail: domenico.siracusa@create-net.org).

\begin{abstract}
We have recently proposed and demonstrated, by means of simulation, the benefits of a simple yet effective cognitive technique to enhance stateless Path Computation Element (PCE) algorithms with the aim of reducing the connection blocking probability when relying on a potentially non up-to-date Traffic Engineering Database (TED). In this paper, we employ that technique, called Elapsed Time Matrix (ETM), in the framework of the CHRON (Cognitive Heterogeneous Reconfigurable Optical Network) architecture and, more importantly, validate and analyse its performance in an emulation environment (rather than in a simulation environment) supporting impairment-aware lightpath establishment. Not only dynamic lightpath establishment on demand has been studied, but also restoration processes when facing optical link failures. Emulation results demonstrate that ETM reduces the blocking probability when establishing lightpaths on demand, and increases the percentage of successful restorations in case of optical link failure. Moreover, the use of that technique has little impact on lightpath setup time and lightpath restoration time, respectively.
\end{abstract}

Keywords - cognition, dynamic lightpath establishment, emulator, outdated database traffic engineering database

\section{Introduction}

Optical transport networks are increasing their complexity due to the use of heterogeneous transmission technologies and the support of services with different requirements [1]. One solution to efficiently control such heterogeneous optical networks comes from the use of cognitive techniques [2]. A cognitive network is defined as "a network with a process that can perceive current network conditions, and then plan, decide, and act on those conditions. The network can learn from these adaptations and use them to make future decisions, all while taking into account end-to-end goals" [2]. Cognitive networks involve the utilization of three types of elements: monitoring elements, which enable the network to be aware of current conditions; software adaptable elements, which enable the network to adapt to changing conditions; and cognitive processes, which learn or make use of past history to improve performance.

Cognitive networks have proved to be an excellent solution for wireless networks [3], and have also been recently proposed for optical networks [4], like the CHRON (Cognitive Heterogeneous Reconfigurable Optical Network) approach $[5,6]$. The CHRON architecture, which will be briefly presented in Section II, is based on a central computation entity, the Cognitive Decision System (CDS) [7], which is responsible of making decisions on how to configure devices and deal with traffic demands, taking into account the current network status. As its name suggests, the CDS leverages on a learning process to improve its performance with acquired experience.

When dealing with a lightpath establishment procedure, the CDS adopts a role similar to that of the Path Computation Element (PCE) [8], which has lately received increasing attention in the optical networking community. The lightpath establishment procedure can be requested by a network user or by the network itself as a part of an optimization process or a restoration process in case of failure. When the CDS receives 
a request to establish a lightpath, it computes a route and a wavelength (or a set of frequency slots if an elastic network is considered) for that optical circuit, according to the current network state. In order to know the network state, the CDS looks up the Topology and Configuration database (TC db), which is a similar concept to the Traffic Engineering Database (TED) in PCE environments. The solution found by the CDS is provided to the source node of the connection, which signals the establishment of the lightpath by means of the RSVP-TE protocol. This action does not necessarily lead to the lightpath establishment because it may happen that one or more resources (assigned by the CDS in its solution) are not actually available. These failures happen because the TC $\mathrm{db}$ (or the TED) is not directly updated by the CDS (or the PCE), but it relies on the OSPF-TE protocol for that aim. This protocol disseminates information about the network topology status and the specific configuration of the devices both at network initialization and after a reservation or release of a physical resource. Therefore, when the CDS makes the decision it must trust the information of the $\mathrm{TC} \mathrm{db}$ even when this information could be outdated. Consequently, the reservation of some lightpaths can fail and the blocking probability of the connections is increased.

This undesirable behaviour is even more important in case of failure. In those situations, not one but many lightpaths may be affected and the restoration processes of all of them are launched immediately. In that moment, the CDS (or PCE) will make wrong decisions as all the requests arrive at nearly the same time and OSPF-TE will not have enough time to update the TC $\mathrm{db}$ between each request.

One possibility to avoid this problem is that the CDS directly updates the TC $\mathrm{db}$ when handling lightpath requests. This solution is similar to the use of a stateful PCE [8]. However, the maintenance and synchronization of the TC $\mathrm{db}$ (or TED) is not trivial, and thus the complexity of the implementation is increased. Moreover, this procedure does not follow the current IETF standards.

Thus, in [9], we proposed a simple yet effective cognitive technique to minimize impact on blocking probability of the TED inaccuracy problem in PCE-based networks. The mechanism, called elapsed times matrix (ETM) heuristic, does not require extensions or modifications to current standardized protocols, and does not introduce additional delays when handling the requests. We demonstrated there that this technique can be used in combination with different routing and wavelength assignment (RWA) algorithms, and we showed its performance by means of a simulation study. However, in that paper the assessment of lightpath quality of transmission was not carried out, and a relevant real-time analysis out of the simulator box is missing.

In this paper, which extends [10], we validate the ETM technique when combined with impairmentaware control methods in the CHRON architecture by means of an emulation environment. The use of an emulator, instead of a simulator, allows us performing a more complete analysis of the response time and the impact of the delays generated by $(i)$ the propagation of control messages, $(i i)$ the insertion/extraction of information in/from the database. Emulation experiments show that the blocking probability cannot be reduced under acceptable values when no technique is used to avoid or reduce the effect of using a non-updated TC db (or TED). Therefore, in very dynamic environments classical RWA methods, which do not apply any strategy to mitigate the effects of outdated databases, could be seriously penalized.However, as can be seen in the study proposed in this paperin our study, the use of the ETM heuristic reduces blocking probability with little impact on lightpath setup time. Moreover, the ETM technique is also very useful in the restoration scenario, where the CDS (or the PCE) must cope with several lightpath restoration processes (one per affected lightpath) and, hence, with several lightpath setup requests, which will be triggered nearly simultaneously. In such a case, it is highly probable that the OSPF-TE will not have time to update the $\mathrm{TC} \mathrm{db}$ between consecutive requests/activations. Although batch processing of these requests are a solution that can be introduced to solve these issues [11, 12], we demonstrate that the use of ETM technique is simple yet effective to overcome these problems.

The paper is organized as follows. Section II and III set the background of this work, by reviewing the CHRON architecture and the ETM proposal, respectively. Then, the CHRON emulator is presented in 
Section IV; Section V describes and analyses the emulation results. In particular, the ETM mechanism is validated in the emulated testbed in two scenarios: lightpath establishment on user demand and lightpath restoration in case of optical link failure. Finally, Section VI summarizes the main conclusions of the paper.

\section{CHRON Architecture}

As previously mentioned, the CHRON architecture is based on a centralized computation entity, the Cognitive Decision System (CDS), supported by a Control and Management System (CMS), which interfaces the CDS to the physical layer (i.e., optical nodes and monitoring devices). By means of the CMS, the CDS retrieves information about the network status (e.g. quality of transmission parameters and resource availability). It also configures the CMS monitoring campaigns over specific network parameters and triggers lightpath setup and teardown mechanisms by means of the GMPLS protocol family (included in the CMS). In this section, we provide an overview of the CHRON architecture, but additional details can be found on [5].

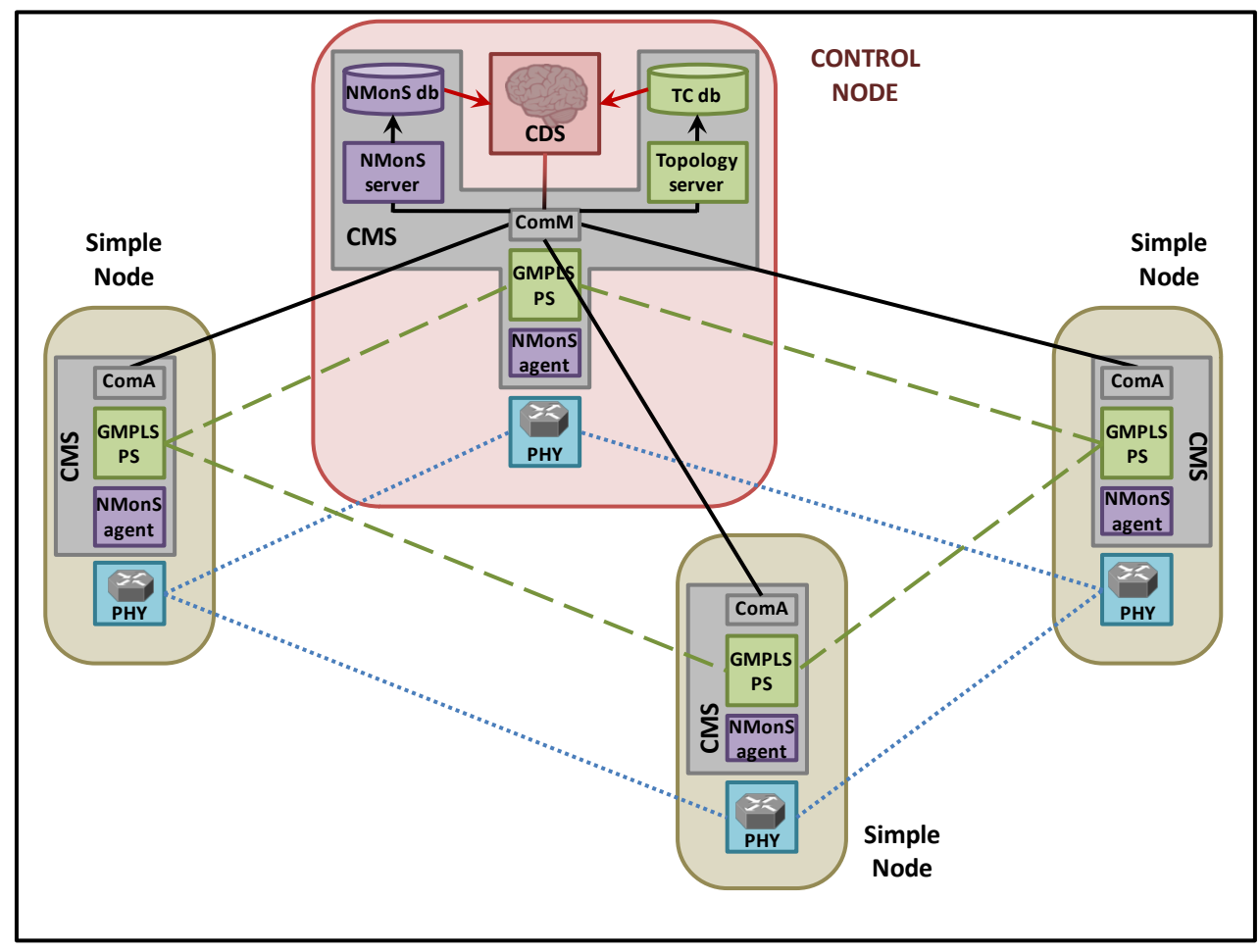

Fig. 1 CHRON architecture in a network composed by four nodes

Fig. 1 shows the high-level CHRON architecture for a network composed by four nodes. More specifically, in this example there are three simple nodes and one control node, which includes the implementation of the CDS.

Each simple node is composed by the following blocks:

- the physical layer module (PHY), which includes the set of different physical devices associated to the node and a physical layer manager handling their configuration and operation;

- the Network Monitoring System (NMonS) agent, which collects information about traffic and the status of the physical devices from the PHY layer; moreover, it provides that information to the NMonS server located in the control node;

- the GMPLS Protocol System (GMPLS PS), which is mainly composed by the OSPF-TE and RSVP-TE protocols, and thus disseminates network information and performs lightpath provisioning; 
- the Communication Agent (ComA), which is the terminal located in each simple node to communicate with the control node. Such channel is used to transmit lightpath setup/teardown requests and responses, and monitoring configuration. Moreover, ComA has an interface (not shown in the figure) allowing an external user to submit requests to the CDS (in XML format) and to receive the response associated to the requested operation.

The control node also includes the first three aforementioned blocks together with:

- the Communication Master (ComM), which is the ComA counterpart at control node side;

- the NMonS server and the NMonS database (NMonS db): they collect and store the information related to the monitored parameters, respectively;

- the Topology Server and the Topology and Configuration database (TC db): the former collects the information related to the network topology and the configuration of the devices (e.g., the wavelength availability in each fiber) and processes the configuration updates provided by the network nodes; that information is stored in the TC $\mathrm{db}$;

- the Cognitive Decision System (CDS), which is the brain of the CHRON architecture and determines how to handle traffic demands or network events, and optimizes network usage and performance by taking into account both the current status of the network and past history. It also instructs the CMS to configure network elements accordingly.

The CDS is involved in various tasks related to network control and optimization. Thus, rather than implementing the whole CDS (the red module in Fig. 1) as a monolithic module, it is divided into different sub-modules each one exploiting cognition to offer a specific functionality (or a set of related functionalities) [7]. In this paper, we focus on the use of three of these sub-modules:

- RWA/RMLSA Module: it solves the routing and wavelength assignment (RWA) problem in traditional fixed-grid networks or the routing, modulation level and spectrum allocation (RMLSA) problem in elastic networks;

- Quality of Transmission (QoT) Estimator Module: it provides estimations (i.e., predictions) about the QoT of new lightpaths to be established in the network as well as the impact on existing connections when undertaking a new one.

- Network Planner \& Decision Maker (NPDM) Module: it receives user requests and handles them by coordinating the operation of the rest of the sub-modules.

\section{Impairment-Aware Lightpath Establishment in Environments with a Potentially Outdated Database: The Elapsed Times Matrix Heuristic}

When the CDS receives a lightpath connection request, either issued by a user or by a network process due to a network failure or a network optimization process, the NPDM sub-module asks the RWA/RMLSA sub-module to search for the set of resources to establish that lightpath. The configuration of each new lightpath provided by the RWA/RMLSA submodule consists of a transmitter, a receiver, a route and a wavelength in the case of a traditional fixed-grid network. During the search, this module interacts with the QoT estimator in order to ensure that the QoT of the new lightpath fulfills Quality of Transmission (QoT) requirements, and that it does not significantly degrades (i.e., below requirements) the QoT of those lightpaths already established in the network. Different tools can be used for this purpose but the cognitive QoT estimator proposed in $[13,14]$ was the method employed in this work mainly due to its speed (important parameter in case of dynamic establishment and lightpath restoration) and accuracy.

When the RWA/RMLSA method searches for the set of resources to establish the new lightpath, it uses the information in the $\mathrm{TC} \mathrm{db}$ to know the availability of resources. As we have previously mentioned, this database is functionally equivalent to the TED in PCE-based environments. As such, the TC $\mathrm{db}$ is not directly updated by the CDS, but relies on the OSPF-TE protocol for that aim. Therefore, the information 
collected there will not be updated until OSPF-TE updates its values. For that reason, the CDS makes decisions based on a database which can be potentially outdated thereby leading to higher blocking probabilities. In case of a network failure, that problem is even more noticeable as not one but many lightpaths should be restored at the same time.

In order to minimize the impact of relying on a potentially outdated database, the Elapsed Times Matrix (ETM) heuristic was proposed in [9]. That heuristic is integrated in the RWA/RMLSA algorithms employed in the CDS, and has the aim of avoiding the selection of wavelengths (or spectrum slots) on fibers, as well as transmitters and receivers, that have been recently assigned to previous lightpath requests, since they will be very probably unavailable (the TC $\mathrm{db}$ has not been updated by OSPF-TE yet, but the RSVP-TE mechanism has already reserved those resources).

The ETM heuristic is based on the use of last time (lt) matrixes and elapsed times (et) matrixes in the CDS.

The last time matrixes contain the instant of time at which a certain resource was assigned for the last time by the CDS. Thus, in the case of a fixed-grid network, one matrix keeps information about wavelength channels $\left(l t_{w c}\right)$, another about transmitters $\left(l t_{t x}\right)$ and another about receivers $\left(l t_{r x}\right)$. In the first matrix, each cell, $l t_{w c}\left(f_{j}, w_{k}\right)$, stores the instant of time at which wavelength $w_{k}$ in fiber $f_{j}$ was assigned by the CDS to a connection for the last time. Similarly, $l t_{t x}\left(n_{s}, t x_{i}\right)$ stores the last time when the CDS assigned transmitter $t x_{i}$ at node $n_{s}$, and $l t_{r x}\left(n_{d}, r x_{j}\right)$ stores the last time at which receiver $r x_{j}$ at node $n_{d}$ was assigned. These matrixes are initialized to zero, and are updated when the CDS makes a decision on which resources to assign to a lightpath.

The elapsed time matrixes contain the elapsed time since the CDS assigned a certain resource for the last time until the current time. One matrix keeps the information about the elapsed time for wavelength channels $\left(e t_{w c}\right)$, another for transmitters $\left(e t_{t x}\right)$ and another for receivers $\left(e t_{r x}\right)$. These matrixes are built each time that a lightpath connection request is received at the CDS by simply subtracting from the current time the value stored in the associated $l t$ matrixes. In this way, for instance, $e t_{w c}\left(f_{j}, w_{k}\right)$, stores the elapsed time since the wavelength $w_{k}$ in fiber $f_{j}$ was assigned by the CDS for the last time. However, if a network resource is not available at that point according to the information in $\mathrm{TC} \mathrm{db}$, the associated cell of the corresponding et matrix is directly set to zero.

When a lightpath setup request between nodes $n_{s}$ and $n_{d}$ is received at the CDS, the elapsed time matrixes are built. Then, an idle transmitter in the source node $n_{s}$ and an idle receiver in the destination node $n_{d}$ are searched. If no transmitter or receiver is available according to the information in the $\mathrm{TC} \mathrm{db}$, the connection is rejected. In the case of more than one idle transmitter in the source node, the transmitter $i$ having a higher value of $e t_{t x}\left(n_{s}, t x_{i}\right)$ is selected for that lightpath. A similar operation is performed to choose the receiver in the destination node, but using the information from the et $t_{r x}$ matrix corresponding to node $n_{d}$. Then, the RWA problem is solved.

The utilization of the elapsed times matrix for wavelength channels can be easily combined with Fixed Routing (FR), Fixed Alternate Routing (FAR) [15] or Adaptive Unconstrained Routing (AUR)-Exhaustive [16] RWA mechanisms, as demonstrated in [9]. In particular, the implementation of ETM in the CHRON emulator uses FAR considering all the possible routes from the source to the destination nodes. Moreover, we assume there is no wavelength conversion in the network. Therefore, the complete algorithm (including ETM) to select a combination of route and wavelength for a lightpath request is:

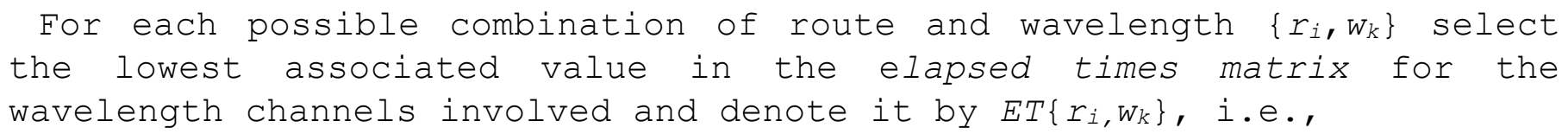


$$
E T\left\{r_{i}, w_{k}\right\}=\min _{j} e t_{w c}\left(f_{j}, w_{k}\right) \quad \text { such that } f_{j} \in r_{i}
$$ \\ From all the possible combinations $\left\{r_{i}, w_{k}\right\}$, select that with the highest value of $E T\left\{r_{i}, w_{k}\right\}$ that fulfils the QoT requirements. If $E T\left\{r_{i}, w_{k}\right\}=0$, the connection is blocked.}

As in the implementation done in the CHRON emulator all possible combinations of routes and wavelengths are considered, the method actually performs an exhaustive search. Thus, with the aim to speed up the process, $E T\left\{r_{j}, w_{k}\right\}$ is calculated in order from the shortest route to the longest one, and when the $E T\left\{r_{j}, w_{k}\right\}$ value for a certain combination of route and wavelength is higher than a user defined threshold, ET required, (high enough to safely assume that the database has been updated by that time regarding the involved resources) and fulfills QoT requirements, this combination is selected as the solution and the search finishes.

When a restoration has to be done due to an optical link failure, no selection of transmitter and receiver should be done as the backup lightpath will use the same ones assigned for the disrupted lightpath. Then, the elapsed time matrix is combined with the AUR-Exhaustive heuristic [16] to solve the RWA problem for the backup lightpath. In this case, an auxiliary graph of the network $\left(G_{k}\right)$ is built for each wavelength $\left(w_{k}\right)$ every time a request arrives at the CDS. The nodes in the auxiliary graphs correspond to the nodes in the physical topology, and two nodes in the auxiliary graph $\left(G_{k}\right)$ are connected by a link if wavelength $w_{k}$ is not used by any lightpath in that link in the physical topology. Once the auxiliary graphs have been built, the lowest cost path in each of them is searched by using the Dijkstra algorithm. The lowest cost path among them is the chosen one for the new connection, together with the corresponding wavelength of that auxiliary graph. When combined with the elapsed times matrix the algorithm is:

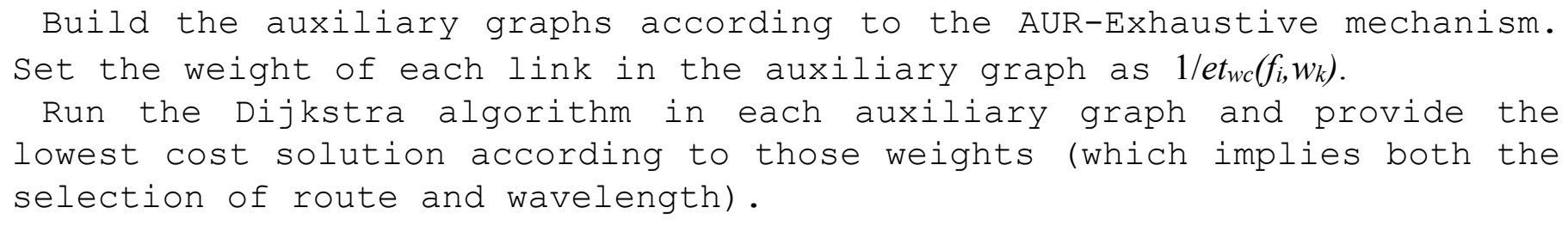

\section{Emulator Description}

The CHRON architecture design presented in Section II has been tailored for the implementation of a working piece of software that emulates the behavior of a cognitive network control and management system. Such emulator, which will be described in this section, has been used to test the ETM method. The emulator provides a more realistic validation of the method than a simulation approach, since it includes a real implementation of the different modules and network protocols and provides a real-time execution.

The modules mainly involved in the operations described in the establishment of a lightpath are the CDS and the GMPLS PS. When the CDS wants to setup a lightpath (either because it has received a request from a client or because it is performing a network optimization process) it communicates the decision to the source node through the communication channel established between them. Such information includes the path with the resources to be reserved, and the parameters to be configured. The source node initiates the reservation procedure by leveraging on a module providing the RSVP-TE protocol [17] (in our implementation RSVP-TE has been extended in order to carry some extra information regarding device configuration). It generates a PATH message storing an Explicit Route Object (ERO) with the path information and the encoded configuration parameters. The PATH message traverses, on the downstream direction (source to destination), every node included in the ERO to check if the requested resources are actually available. The information related to the configuration of devices (i.e., transmitters, receivers, switch ports, amplifiers, etc.) is extracted from the packet and forwarded to the local resource manager module (called CAC/RM, since it is also in charge of channel access control), which performs such 
consistency check. If the latter is successful, the PATH message is sent to the next node contained in the ERO, up to the destination; otherwise, a PATH Error message is generated and sent backward to the source node, which informs the CDS by means of the above mentioned communication channel. If the downstream check is successful, a RESV message is triggered and goes through the upstream (destination to source) direction in order to carry out the configuration of the nodes. Such operation is actually performed by the CAC/RM, which then takes two actions. The first one is to inform the RSVP-TE module that the configuration of the node has been done successfully, such that the RESV message can be forwarded to the next node in the up direction. When all devices have been configured (i.e., including the source node), the reservation process is complete. We have added a feedback message for the CDS included into the answer to its initial request. The second action taken by the CAC/RM is to inform the OSPF-TE module about the changes made on the configuration of the current node. In each node, within the homonym module, the OSPF-TE protocol [18] is implemented. The notifications received from $\mathrm{CAC} / \mathrm{RM}$ are encoded into Link State Advertisements (LSAs, extended since they carry additional information about device configuration) in OSPF-TE packets and then shared among the OSPF-TE modules of the other network nodes, strictly following the protocol. Whenever an LSA arrives at the Control Node, the update is forwarded to the Topology Server in order to be saved in the TC db and used for future evaluations.

From the implementation point of view (with respect to the architecture shown in Fig. 1), the software implementing the modules of the CDS has been written in $\mathrm{C}++$, tested in an OMNeT++-based simulator and then exported for the emulator. It talks via XML messages through socket connection with the rest of the architecture. The core software of the GMPLS PS is the Dragon GMPLS framework [19], an opensource $\mathrm{C} / \mathrm{C}++$ software relying on GNU Zebra routing software [20]. It provides a suite of software modules implementing the main components of a GMPLS control plane (including OSPF-TE and RSVPTE). In addition, a set of Python scripts (Control Plane Scripts, CPS) have been implemented in order to ease the interfacing of the GMPLS PS modules with the rest of the architecture: this approach minimizes the modifications to the original Dragon modules and simplifies the integration and the communication with other modules, like the Communication modules (Master and Agent) and the CAC/RM, which have been implemented in Python, too. The two servers (NMonS and Topology Server) are also Python scripts, while the two databases are defined in SQL and have been created with postgreSQL. For the experiments carried out in this work, we have improved the efficiency of the emulator: with respect to the monitored values we are only interested in alarms and not on the historical progression of the values. Therefore, we have disabled (with a revertible operation) the NMonS database and stored the information related to alarms in the TC database. By doing so, the CDS needs to read just from one database, instead of querying two different databases (please note that the information related to alarms is negligible, so managing a second database was not really meaningful in this case). Hence, NMonS server is replaced by the Topology Server; the latter writes on the TC database both the messages received from the OSPF-TE module and the NMonS agent related to network configuration and managed monitors, respectively. Finally, the data plane is emulated: the physical layer is restricted to a limited amount of functionalities, mainly related to the emulated configuration of the devices (i.e., transmitter, receivers, and OXCs). For this reason, we have combined the NMonS agent and the PHY modules into a single module emulating the behaviour of the physical devices during the network provisioning procedures.

Finally, with respect to the hardware used in the implementation of the emulator, the software is running on a single host machine, a server DELL PowerEdge R310 with 32GB RAM equipped with a 4-core Intel Xeon $\mathrm{X} 3480,3.06 \mathrm{GHz}$ and $2 \mathrm{~TB}$ hard disk. The control node and the simple nodes are running in guest virtual machines, while the CDS modules and the TC $\mathrm{db}$ are installed in the host machine. There are different reasons behind this choice. First of all, from an architectural point of view, it is plausible that in a real implementation the CDS would be installed in a dedicated machine. Moreover, the computations carried out by the CDS are memory-consuming and may require a lot of computational capacity, thus it is better to run them in the host machine, since it has more available resources. 


\section{Emulation Results}

\subsection{Description of the experiment}

In order to test the advantages of using the ETM method in the CHRON architecture, the emulator previously described has been employed. The 14-node Deutsche Telecom network has been emulated assuming that each cable consists of one fiber per direction, and each fiber is configured with 32 fixed-grid wavelength channels.

Two different experiments will be presented. In the first one, users request the establishment of lightpaths according to a Poisson process. Thanks to these tests, it will be shown that the blocking probability of the network decreases when the ETM mechanism is employed. In the second one, the advantages of ETM to restore lightpaths when facing optical link failures will be shown. In that experiment, many restoration messages arrive at the CDS at almost the same time. Thanks to the use of ETM, the probability of successful restoration increases.

\subsection{Advantages of using ETM in an on-demand lightpath establishment scenario}

In this test, emulated users send lightpath setup requests (up to 3,000) to the CDS according to a Poisson process. The source and the destination of each lightpath are randomly selected according to a uniform distribution. Statistics are retrieved on whether the connection is successfully established or not. Moreover, in the latter case we also get track of where the blocking of the connection took place:

- CDS BP: the CDS does not find an available combination of route and wavelength to establish the lightpath satisfying QoT requirements, and without significantly degrading the QoT of those lightpaths previously established.

- CMS BP: the CDS may make decisions on outdated information and the reservation made by RSVP-TE fails trying to reserve network resources which are already in use.

Moreover, the use of an emulator has also enabled collecting meaningful results on lightpath setup time (in contrast with simulation studies).

Regarding RWA mechanisms, two different options have been explored. On the one hand, the FAR method has been used together with the ETM mechanism, as described in Section III. In particular, all the possible routes from the source to the destination nodes are considered. Hence, the threshold to accelerate the ETM mechanism (ET $\left.T_{\text {required }}\right)$ has been set to 50 seconds. On the other hand, when the ETM method is not employed, FAR has also been used (again considering all the possible source-destination routes) and first-fit was used as the wavelength assignment mechanism [15]. This second option serves as a benchmark to compare the performance of the ETM technique.

First of all, in Fig. 2, we show the blocking probability obtained with and without using the ETM technique. Note that we show the total blocking probability (BP), and also split it in its two components (as previously mentioned): blocking probability due to the CDS (CDS BP) and blocking probability due to the CMS (CMS BP). For low traffic loads, the main contribution to the total blocking probability comes from the CMS system (i.e., due to having relied on outdated information when deciding the combination of route and wavelength), while for high traffic loads, it is mainly due to the CDS (i.e., due to lack of network resources). 


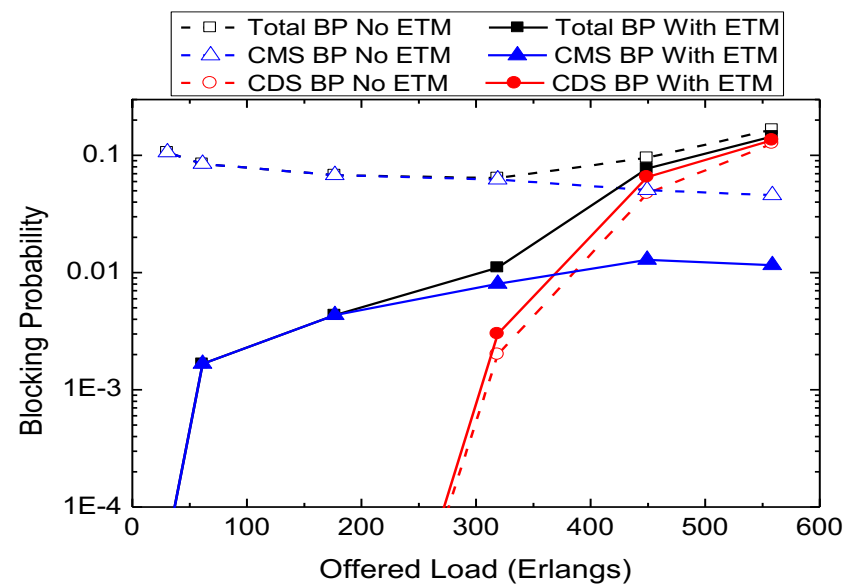

Fig. 2 Blocking probability (BP) obtained with and without ETM

Fig. 2 also shows that the total blocking probability is reduced when ETM is employed. The reason is that ETM avoids the use of resources recently assigned to other connections (if possible). That leads to a reduction on the blocking probability induced by the CMS (i.e., that due to relying on an outdated database), without causing a significant increment in the blocking probability of the CDS. The slight increment in the blocking probability due to the CDS when ETM is used comes out because the CDS can decide to employ longer routes in order to avoid using resources recently assigned, thus requiring more network resources.

On the other hand, it is worth to note that due to the method we have used to increase the traffic load in the emulator, which consists in extending the duration of the connections, the network state changes less frequently, and for that reason, the CMS blocking probability (which is due to relying on an outdated TC $\mathrm{db}$ ) may decrease with increasing load. (This effect of reduction of the blocking probability due to outdated databases when increasing the load by augmenting the duration of the connections has also been reported in other works on distributed lightpath establishment, e.g. [21, 22]).

Fig. 3 shows the average time that the CDS requires to assign resources to an incoming lightpath request, i.e., from the moment in which the lightpath request arrives at the CDS until the time in which the CDS notifies the CMS with the configuration of the set of resources required to establish the lightpath (or to the user if no resources are available for the connection).

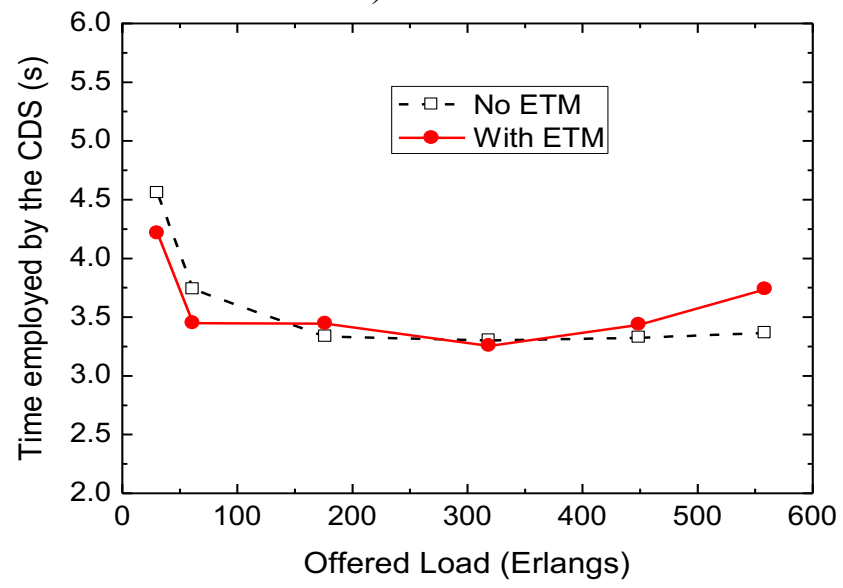

Fig. 3 Time required by the CDS to process a request

Fig. 3 shows that the CDS requires around 3 or 4 seconds to find a solution for the traffic loads tested. The reason for this timing is that not only the set of transmitter, receiver, route and wavelength has to be found by the CDS (together with the assessment of the QoT), but it must also decode and compose XML 
messages and query the $\mathrm{TC} \mathrm{db}$, and finally, handle and process other messages (also requiring CDS functionalities) arriving at the CDS meanwhile. On the other hand, Fig. 3 also shows that the use of ETM does not really increase the time that the CDS requires to determine the resources to be assigned to a lightpath.

Then, Fig. 4 shows the response time to the user in case of a positive establishment, i.e., the time elapsed from the instant in which the user makes the request to the CDS until when the user is notified of the success of the connection setup, that is, the lightpath setup time. The time to provide a response to the user when the establishment fails is shown in Fig. 5. In the latter case, we make an additional distinction by showing the response time when blocking is due to the CDS (either due to the lack of resources or low QoT) or due to the CMS (due to a failure in the reservation process).

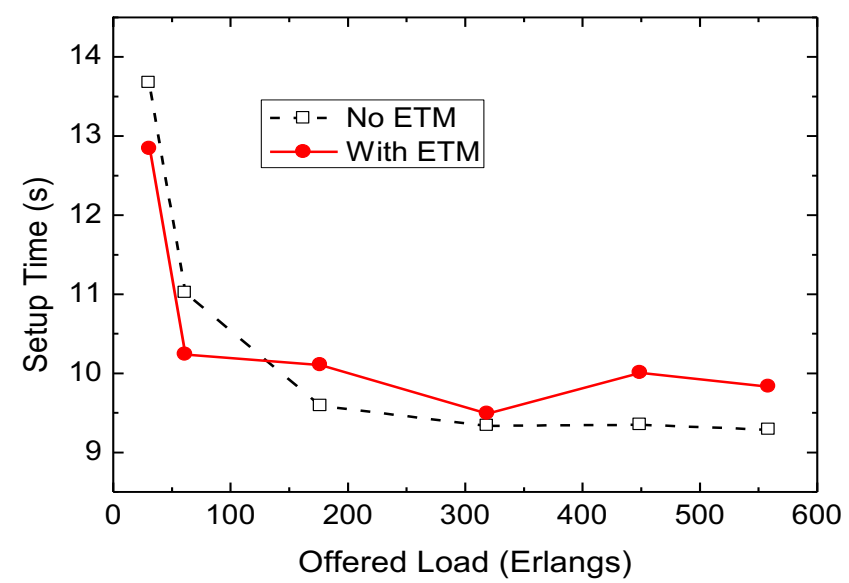

Fig. 4 Response time to the user (setup time) in case of a positive establishment

Fig. 4 shows that the network requires more time for lightpath setup when ETM is used, as it usually leads to using longer routes, which means that RSVP-TE needs more time to establish the lightpath since it has to configure more nodes (also, longer propagation delays could be experienced). However, these differences in time are lower than 5\% for all network loads. It is important to remark that the time required establishing a lightpath in the DT network in the analysed scenarios is between 10 and 14 seconds. Since the time required by the CDS to process a request is around 3 or 4 seconds (see Fig. 3), it can be seen that most of setup time is due to the use of RSVP-TE to reserve and configure the equipment in the network and activate the transmitter and receiver.

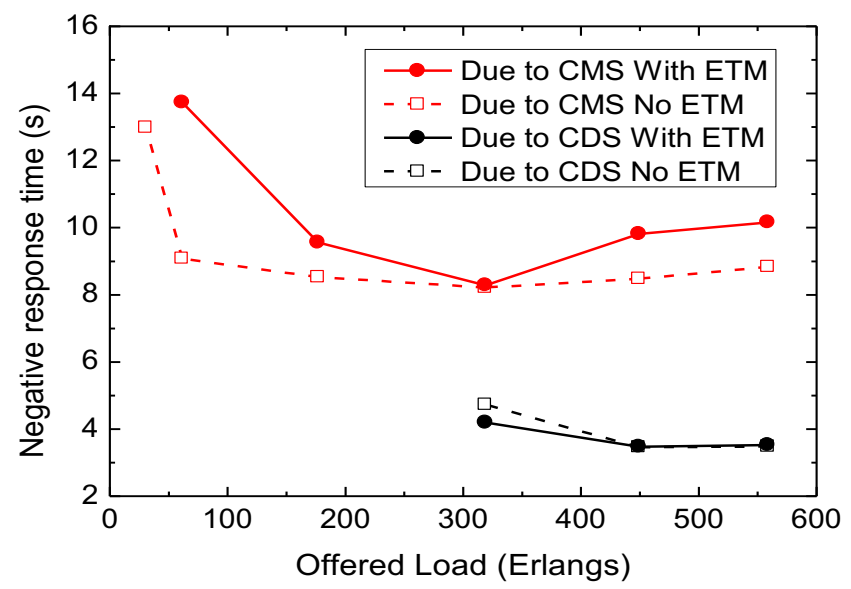

Fig. 5 Response time to the user in case of a failed establishment

Finally, as shown in Fig. 5, the time elapsed until the user is notified about the failure in the establishment of a lightpath is obviously lower when it happens in the CDS process than when it occurs in 
the CMS process. (Please note that for low network loads, the time is not represented when the blocking is due to the CDS because no connections were blocked due to the CDS. The same issue happens for the lowest network load when ETM is used and the blocking is due to the CMS.)

In conclusion, the studies performed with the emulator to evaluate the use of ETM when establishing ondemand lightpaths have shown that it reduces the blocking the blocking probability in networks with potentially outdated TED without any significant increasing in the response time of the network.

\subsection{Advantages of using ETM in a restoration scenario}

We now analyse the performance of ETM in a restoration scenario due, for instance, to optical link failures. In this scenario, the CDS receives many requests for lightpath restoration (one per affected lightpath) nearly simultaneously. Rather than handling these requests jointly, which would imply introducing a new control mechanism in the network, we analyse the capacity of ETM of directly handling these nearly simultaneous requests and overcoming the associated issue of having an outdated database.

As in the previous section, emulated users send lightpath setup requests to the CDS according to a Poisson process. The network is initially empty, so a warm-up period is considered until the average number of connections in the network is approximately that corresponding to the injected traffic load. At that point, i.e., when the network has reached a stationary state, an optical link failure affecting the two fibers of a randomly chosen link is emulated. The restoration process is launched and, when it finishes, the fiber is repaired and the network continues its operation until reaching a stationary state. Then, another random optical link fails, and the process is repeated until having statistically meaningful results. Statistics are retrieved on successfully/un-successfully restored lightpaths, and on the mean restoration time in case of failure. The method followed by the CDS to restore a lightpath is based on an exhaustive search method, as explained in Section III.

Fig. 6, shows the number of affected lightpaths per optical link failure as a function of the offered load, with and without ETM. When ETM is used, the number of affected lightpaths is slightly higher. This is due to the fact that the blocking probability when establishing primary lightpaths is lower when the ETM heuristic is used (as shown in Fig. 2) and, therefore, the number of lightpaths established in the network is higher. For that reason, the average number of affected lightpaths when an optical link failure happens is higher, and thus the average number of lightpaths that must be restored in those cases is also higher.

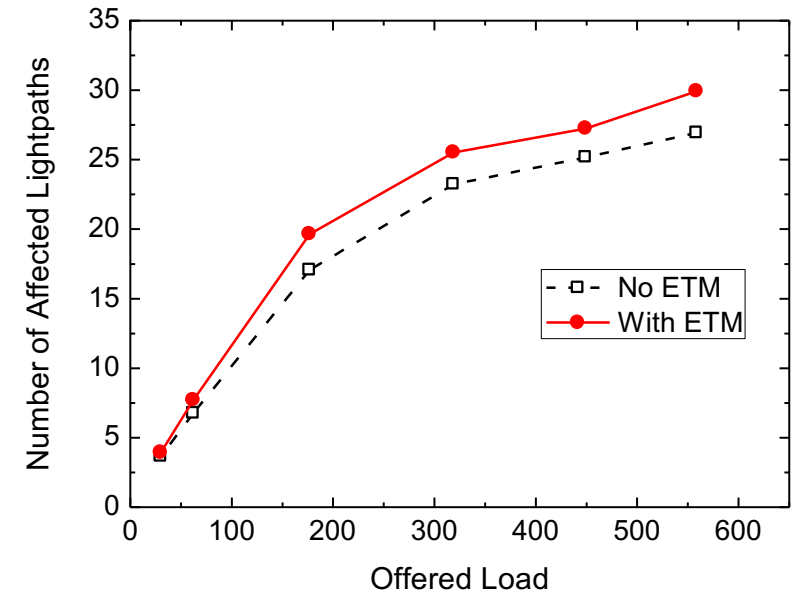

Fig. 6 Number of affected lightpaths in function of the offered network load

Fig. 7 shows the percentage of successful lightpath restorations as a function of the traffic load. Moreover, it also shows the percentage of successful restoration according to the CDS, i.e., those lightpaths that could be restored according to the CDS as it finds a set of resources to establish the backup lightpath. Finally, Fig. 7 also shows the percentage of successful restorations due to the CMS, i.e., the percentage of lightpaths that are finally established in the network compared with the ones provided by the CDS. (The 
percentage of successful lightpath restorations is, therefore, the product of these values, i.e., the success rate of the CDS and the CMS).

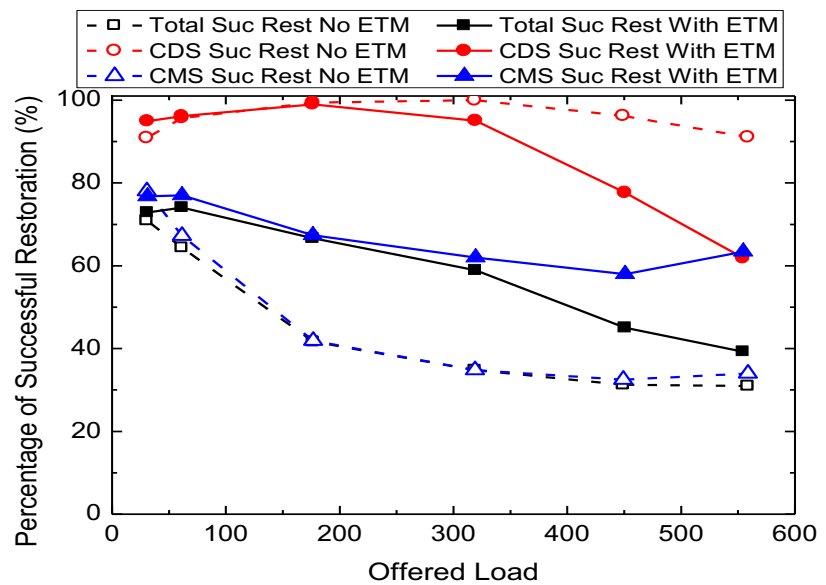

Fig. 7 Percentage of affected lightpaths successfully recovered, and the success percentage of the CDS and the CMS, with and without using ETM

Results from Fig. 7 show that despite the fact that more lightpaths should be restored in each optical link failure when ETM is used (as shown in Fig. 6), the total percentage of successful restorations when ETM is used is higher than when it is not used. This effect is more significant in medium loads, where the improvement reaches $30 \%$.

On the other hand, when the success rate of the CDS is analysed, it can be seen that the CDS finds resources to successfully restore more than $90 \%$ of the affected lightpaths, for low and medium traffic loads, independently of whether ETM is used or not. However, when the network is highly loaded, the success ratio of the CDS is lower if ETM is used. This is due to the fact that ETM leads to employing longer routes (to avoid the use of resources that have been recently assigned) and thus requires more resources. However, that strategy of avoiding the use of resources that have been recently assigned leads to a higher success ratio of the CMS, and lastly to a higher total percentage of successful restorations.

Finally, Fig. 8 shows the mean time required to restore a lightpath with and without using ETM. That graph shows that the restoration time is similar independently of using or not the ETM technique. For low offered loads, the restoration time increases due to the fact that longer restoration routes can be employed (if needed), as there are more available resources.

In conclusion, when many lightpaths should be restored due to a network failure, the ETM technique increases the probability of successful restoration without causing a penalty in the time required to perform the restoration.

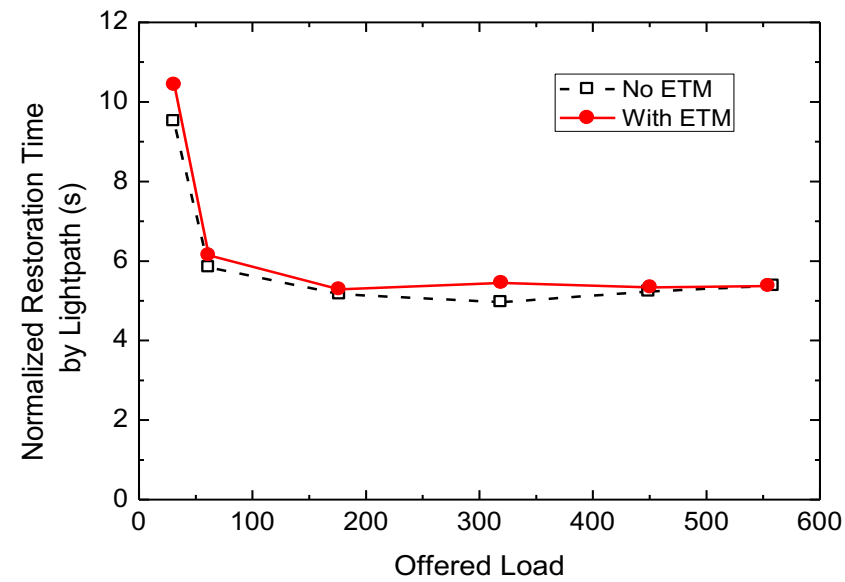

Fig. 8 Normalized restoration time dedicated to recover each lightpath 


\section{Conclusion}

Outdated TED may affect network performance when solving the RWA problem in environments where the TED update is done by means of network protocols like OSPF-TE. In order to minimize the impact of this issue, the ETM technique was proposed and validated by means of simulation [9]. Such technique avoids the selection of resources that have been recently assigned to other lightpaths. In this paper we have enhanced that work by incorporating the ETM technique into the impairment-aware and cognitive CHRON architecture, and, mainly, by validating and studying its performance in two test-cases within an emulation environment. Firstly, it is shown that the ETM method can be effectively used to reduce the blocking probability in the establishment of on-demand lightpaths. Secondly, emulation results showed that using ETM it is possible to increase the percentage of successful restoration in case of an optical link failure. Moreover, the use of this technique does not affect the time required to perform both operations.

\section{Acknowledgment}

This work has been funded by the European Com- munity's Seventh Framework Programme [FP7/20072013] CHRON project (Cognitive Heterogeneous Reconfigurable Optical Network) under grant agreement no 258644, http://www.ict-chron.eu, and Span- ish Ministry of Science and Innovation (TEC2010-21178C02-02 and TEC2014-53071-C3-2-P).

\section{.References}

[1] Tomkos, I., Angelou, M., Durán, R.J., de Miguel, I., Lorenzo, R.M., Siracusa, D., Salvadori, E, Tymecki, A., Ye, Y.: Next generation flexible and cognitive heterogeneous optical networks. The Future Internet - Future Internet Assembly 2012: From Promises to Reality, pp. 225-236. Springer, (2012).

[2] Thomas, R.W., Friend, D.H., DaSilva, L.A., MacKenzie, A.B.: Cognitive networks: adaptation and learning to achieve endto-end performance objectives. IEEE Communications Magazine, vol.44, no.12, pp.51-57, (2006). doi: 10.1109/MCOM.2006.273099.

[3] Mahmoud, Q.H., Cognitive networks: Towards Self-Aware networks, John Wiley \& Sons, Ltd. (2007).

[4] de Miguel, I., Durán, R.J., Jiménez, T., Fernández, N., Aguado, J.C., Lorenzo, R.M., Caballero, A., Tafur Monroy, I., Ye, Y., Tymecki, A., Tomkos, I., Angelou, M., Klonidis, D., Francescon, A., Siracusa, D., Salvadori, E.: Cognitive dynamic optical networks [invited]. IEEE/OSA Journal of Optical Communications and Networking, vol.5, no.10, pp.A107-A118, (2013). doi: 10.1364/JOCN.5.00A107.

[5] Caballero, A., Borkowski, R., de Miguel, I., Durán, R.J., Aguado, J.C., Fernández, N., Jiménez, T., Rodríguez, I., Sánchez, D., Lorenzo, R.M., Klonidis, D., Palkopoulou, E., Diamantopoulos, N., Tomkos, I., Siracusa, D., Francescon, A., Salvadori, E., Ye, Y., López J., Pittalà, F., Tymecki, A., Tafur, I.: Cognitive, Heterogeneous and Reconfigurable Optical Networks: The CHRON Project, J. Lightwave Technol. 32, 2308-2323 (2014).

[6] EU FP7 CHRON project, [Online] http://www.ict-chron.eu (2015). Accessed 26 March 2015.

[7] Durán, R.J., De Miguel, I., Sánchez, D., Fernández, N., Jiménez, T., Aguado, J.C., Yedugundla, V.K., Angelou, M., Merayo, N., Fernández, P., Atallah, N., Lorenzo, R.M., Francescon, A., Tomkos, I., Abril, E.J.: A cognitive decision system for heterogeneous reconfigurable optical networks. Future Network \& Mobile Summit (2012).

[8] Farrel, A., Vasseur, J.-P.: A Path Computation Element (PCE)-Based Architecture,” IETF RFC 4655. (2006).

[9] Rodríguez, I., Durán, R.J., Siracusa, D., de Miguel, I., Francescon, A., Aguado, J.C., Salvadori, E., Lorenzo, R.M.: Minimization of the impact of the TED inaccuracy problem in PCE-based networks by means of cognition. ECOC (2013). doi: 10.1049/cp.2013.1487.

[10] Fernández, N., Durán, R.J., Siracusa, D., Francescon, A., de Miguel, I., Rodríguez, I., Aguado, J.C., Salvadori, E., Lorenzo, R.M.: Experimental assessment of a cognitive mechanism to reduce the impact of outdated TEDs in optical networks. ICOCN (2014). doi: 10.1109/ICOCN.2014.6987070

[11]Lee, Y., Le Roux, JL., King, D., Oki, D.: Path Computation Element Communication Protocol (PCEP) Requirements and Protocol Extensions in Support of Global Concurrent Optimization. IETF RFC 5557, July 2009.

[12] Martínez, R., Castro, A., Casellas, R., Muñoz, R., Velasco, L., Vilalta, R., Comellas, J.: Experimental validation of dynamic restoration in GMPLS-controlled multi-layer networks using PCE-based global concurrent optimization. in Proc. OFC (2013), paper OW4G.3, http://dx.doi.org/10.1364/OFC.2013.OW4G.3

[13] Jiménez, T., Aguado, J.C., de Miguel, I., Durán, R.J., Angelou, M., Merayo, N., Fernández, P., Lorenzo, R.M., Tomkos, I., Abril, E.J.: A Cognitive Quality of Transmission Estimator for Core Optical Networks. Journal of Lightwave Technology, vol.31, no.6, pp.942,951. (2013). doi: 10.1109/JLT.2013.2240257 
[14] Caballero, A., Aguado, J.C., Borkowski, R., Saldaña, S.,, Jiménez, T., de Miguel, I., Arlunno, V., Durán, R.J., Zibar, D., Jensen, J., Lorenzo, R.M., Abril, E.J., Monroy, I.: Experimental demonstration of a cognitive quality of transmission estimator for optical communication systems. Opt. Express 20, B64-B70 (2012).

[15]Zang, H., Jue, H.P. : A review of routing and wavelength assignment approaches for wavelength-routed optical WDM networks. Optical Networks Magazine, pp. 47-60, (2000).

[16] Mokhtar, A., Azizoglu, M.: Adaptive Wavelength Routing in All-Optical Networks. IEEE/ACM Transactions on Networking vol. 6, no. 2, pp. 197, (1998). DOI: 10.1109/90.664268

[17] Awduche, D., Berger, L., Gan, D., Li, T., Srinivasan, V., Swallow, G.: RSVP-TE: Extensions to RSVP for LSP Tunnels. IETF RFC 3209, (2001).

[18] Katz, D., Kompella, K., Yeung, D.: Traffic Engineering (TE) Extensions to OSPF Version 2. IETF RFC 3630. (2003).

[19] GMPLS website [Online], available: http://dragon.east.isi.edu/twiki/bin/view/DRAGON/WebHome (2015) Accessed 26 March 2015

[20] GNU Zebra website

[Online] Available: http://www.gnu.org/software/zebra/ (2015). Accessed 26 March 2015

[21] Aragón, V.M., de Miguel, I., Durán, R.J., Merayo, N., Aguado, J.C., Fernández, P., Lorenzo, R.M., Abril, E.J.: A New Algorithm for the Distributed RWA Problem in WDM Networks Using Ant Colony Optimization. in Optical Network Design and Modeling., pp. 299-308. Springer, (2007).

[22]Lu, K., Xiao, G., Chlamtac, I.: Analysis of blocking probability for distributed lightpath establishment in WDM optical networks. IEEE/ACM Trans. on Networking., vol. 13, no. 1, pp. 187-197, (2005). 